\title{
Perturbations of the anti-ageing hormone Klotho in patients with type 1 diabetes and microalbuminuria
}

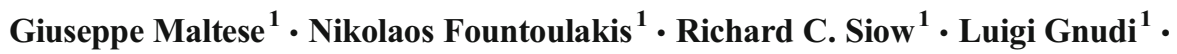 \\ Janaka Karalliedde ${ }^{1}$
}

Received: 21 September 2016 / Accepted: 9 December 2016 /Published online: 13 February 2017

(C) The Author(s) 2017. This article is published with open access at Springerlink.com

\begin{abstract}
Aims/hypothesis Patients with type 1 diabetes and microalbuminuria are at high risk of cardiovascular disease (CVD) and end-stage renal disease. Soluble Klotho is an anti-ageing circulating hormone involved in phosphate metabolism and vascular homeostasis through protective effects on the endothelium and antioxidant actions. The role of soluble Klotho in patients with type 1 diabetes and microalbuminuria is unknown.

Methods In a cross-sectional single-centre study we evaluated the levels of circulating serum soluble Klotho in 33 participants with type 1 diabetes and a history of microalbuminuria (receiving renin-angiotensin system [RAS] inhibitors) and 45 participants with type 1 diabetes without a history of microalbuminuria (not receiving RAS or other antihypertensive drugs). All participants had an eGFR $>45 \mathrm{ml} / \mathrm{min}$, duration of diabetes $>20$ years and no history of CVD. Serum soluble Klotho levels were measured by a validated immunoassay.

Results Participants with microalbuminuria had significantly lower levels of serum Klotho compared with those without microalbuminuria (median [interquartile range], 659.3 $[525.3,827.6]$ vs $787.7[629.5,1007] ; p=0.023)$. This difference persisted after adjustment for variables including
\end{abstract}

Electronic supplementary material The online version of this article (doi:10.1007/s00125-017-4219-1) contains peer-reviewed but unedited supplementary material, which is available to authorised users.

Giuseppe Maltese

giuseppe.maltese@kcl.ac.uk

1 Unit for Metabolic Medicine, Cardiovascular Division, Faculty of Life Sciences and Medicine, King's College London, 150 Stamford Street, London SE1 9NH, UK age and eGFR. In a subgroup of 30 individuals with and without microalbuminuria, other markers of phosphate balance were not significantly different.

Conclusions/interpretation In individuals with type 1 diabetes, microalbuminuria is associated with soluble Klotho deficiency. Further studies are required to determine whether soluble Klotho is causally related to the development of cardio-renal disease in type 1 diabetes.

Keywords Microalbuminuria $\cdot$ Soluble Klotho $\cdot$ Type 1 diabetes

$\begin{array}{ll}\text { Abbreviations } \\ \text { ACR } & \text { Albumin to creatinine ratio } \\ \text { CVD } & \text { Cardiovascular disease } \\ \text { DKD } & \text { Diabetic kidney disease } \\ \text { FGF-23 } & \text { Fibroblast growth factor-23 } \\ \text { MA } & \text { Microalbuminuria } \\ \text { MA }^{+} & \text {Presence of microalbuminuria } \\ \text { MA }^{-} & \text {Normoalbuminuria } \\ \text { PTH } & \text { Parathyroid hormone } \\ \text { RAS } & \text { Renin-angiotensin system }\end{array}$

\section{Introduction}

Microalbuminuria (MA) is one of the earliest manifestations of diabetic kidney disease (DKD) in type 1 diabetes [1]. MA, a marker of endothelial dysfunction, is associated with a higher risk of cardiovascular disease (CVD) morbidity and mortality [1]. Increased oxidative stress and endothelial dysfunction are key events contributing to the pathogenesis of DKD and CVD [1].

Klotho is a nephroprotective transmembrane protein predominantly expressed in the renal tubules and implicated 
in regulating phosphate metabolism, together with fibroblast growth factor-23 (FGF-23) [2]. A circulating form of Klotho, named soluble Klotho (resulting from a proteolytic cleavage of the transmembrane protein), is detectable in the circulation and has been demonstrated to maintain vascular homeostasis through antioxidant properties [2]. In vivo, soluble Klotho deficiency is accompanied by activation of the renin angiotensin system (RAS) and endothelial dysfunction [2]. Individuals with type 2 diabetes and an eGFR $>60 \mathrm{ml} / \mathrm{min}$ have reduced tissue levels of Klotho compared with individuals with $\operatorname{IgA}$ nephropathy [3]. ESM Table 1 summarises human and in vivo studies where Klotho levels have been associated with DKD. In rodent models of type 1 diabetes, renal expression of Klotho is reduced [3]. To date there is a lack of information on the relationship between circulating soluble Klotho and MA in patients with type 1 diabetes.

\section{Methods}

This study included 78 individuals with type 1 diabetes attending the diabetes outpatient clinic at Guy's Hospital, London, UK. Of these, 33 had microalbuminuria $\left(\mathrm{MA}^{+}\right)$and 45 had normoalbuminuria $\left(\mathrm{MA}^{-}\right)$. All study participants gave informed consent and investigations were approved by the responsible ethics committee. Clinical details and biochemical measurements for the cohort are shown in Table 1. For measurement of serum Klotho, blood samples were immediately centrifuged at $1500 \mathrm{~g}$ at $4^{\circ} \mathrm{C}$ for $10 \mathrm{~min}$ and the supernatant fractions were stored at $80^{\circ} \mathrm{C}$ (for $<24$ months) with no freeze-thaw cycles before analysis. This approach has been shown not to impact on the sensitivity of the assay used in this study [4]. All samples were assayed in duplicate using an immunoassay kit (Immuno-Biological-Laboratories, Hamburg, Germany) [4-6].

All participants had an eGFR $>45 \mathrm{ml} / \mathrm{min}$, duration of diabetes $>20$ years and no history of CVD. Participants in the $\mathrm{MA}^{+}$group had a positive history of MA (more than two early morning urine albumin to creatinine ratio (ACR) measurements $\geq 3.0 \mathrm{mg} / \mathrm{mmol}$ ), confirmed diabetic retinopathy and were receiving RAS inhibitor treatment. The participants in the $\mathrm{MA}^{-}$group were not receiving any RAS inhibitor treatment or any other antihypertensive agents.

An investigator blinded to MA status performed the soluble Klotho measurements. Urine ACR was measured from morning urine samples. Urine creatinine (Jaffe reaction) and albumin concentrations were measured by immunoturbidimetry, serum total cholesterol (enzymatic colorimetry) and creatinine (rate reaction method) levels were measured using a Cobas Mira Plus analyser (Roche Diagnostics, Rotkreuz, Switzerland) [6]. $\mathrm{HbA}_{1 \mathrm{c}}$ was measured by boronate affinity HPLC (Primus CLC330, Kansas City, MO, USA). eGFR was determined using the Chronic Kidney Disease Epidemiology Collaboration Formula (CKD-EPI) [7].

Descriptive statistics were used for the analysis of demographic and clinical features. Participants with and without MA were compared by an unpaired $t$ test (for continuous normally distributed variables), Mann-Whitney test (for continuous variables not normally distributed) and $\chi^{2}$ test (for categorical variables). Testing for normality was performed using the Shapiro-Wilk test. Since soluble Klotho levels and ACR were not normally distributed, their values were $\log$ transformed (base $e$ ) for Pearson correlation analysis and multivariate logistic regression analysis. Multivariate logistic regression analysis was performed to evaluate if the
Table 1 Comparison of the clinical and biochemical features of 78 type 1 diabetes participants with $\left(\mathrm{MA}^{+}\right)$and without $\left(\mathrm{MA}^{-}\right)$ microalbuminuria

\begin{tabular}{|c|c|c|c|}
\hline Variable & $\mathrm{MA}^{+}(n=33)$ & $\mathrm{MA}^{-}(n=45)$ & $p$ value \\
\hline Age, years & $54.4 \pm 11.6$ & $43.3 \pm 9.6$ & $<0.001$ \\
\hline Sex, \% male $(\mathrm{M} / \mathrm{F})$ & $70(23 / 10)$ & $44(20 / 25)$ & 0.03 \\
\hline Retinopathy, \% & 100 & 100 & NS \\
\hline Use of RAS inhibitors, $\%$ & 100 & 0 & $<0.001$ \\
\hline Use of statins, \% (n) & $70(23)$ & $29(13)$ & $<0.001$ \\
\hline Diabetes duration, years & $32.7 \pm 10.2$ & $29.5 \pm 9.7$ & 0.21 \\
\hline $\mathrm{HbA}_{1 \mathrm{c}}, \%$ & 7.7 & 7.9 & 0.30 \\
\hline $\mathrm{HbA}_{1 \mathrm{c}}, \mathrm{mmol} / \mathrm{mol}$ & $60.9 \pm 10.7$ & $63.0 \pm 10.0$ & \\
\hline $\mathrm{SBP}, \mathrm{mmHg}$ & $132.6 \pm 12.4$ & $126.3 \pm 13.1$ & 0.06 \\
\hline DBP, mmHg & $72.3 \pm 9.2$ & $74.8 \pm 8.5$ & 0.26 \\
\hline $\mathrm{eGFR}, \mathrm{ml} / \mathrm{min}$ & $90.2 \pm 21.7$ & $100.2 \pm 20.4$ & 0.054 \\
\hline $\mathrm{ACR}, \mathrm{mg} / \mathrm{mmol}^{\mathrm{a}}$ & $1.1(0.5,8.1)$ & $0.7(0.5,1.05)$ & 0.013 \\
\hline Soluble Klotho, pg/ml ${ }^{\mathrm{a}}$ & $659.3(525.3,827.6)$ & $787.7(629.5,1007)$ & 0.023 \\
\hline
\end{tabular}

All data are mean \pm SD unless otherwise stated

${ }^{\text {a }}$ Median (interquartile range)

DBP, diastolic BP; SBP, systolic BP 
significant association between MA status and Klotho levels persisted when adjusted for other variables. Data are given as mean $\pm \mathrm{SD}$, percentage for categorical variables, or median and interquartile range for variables not normally distributed. A two-tailed $p$ value $<0.05$ was considered significant. Statistical analyses were performed with SPSS version 19.0 (SPSS, Chicago, IL, USA).

\section{Results}

Participants in the $\mathrm{MA}^{+}$group were older $(54.4 \pm 11.6$ vs $43.3 \pm 9.6$ years), more frequently male (70\% vs $44 \%)$ and more frequently on statin treatment ( $70 \%$ vs $29 \%$ ) compared with the $\mathrm{MA}^{-}$group $(p<0.05$ for all; Table 1$) . \mathrm{MA}^{+}$ participants also had higher systolic BP $(132.6 \pm 12.4 \mathrm{vs}$ $126.3 \pm 13.1 \mathrm{mmHg})$, lower eGFR $(90.2 \pm 21.7 \mathrm{vs}$ $100.2 \pm 20.4 \mathrm{ml} / \mathrm{min}$ ) and lower levels of serum Klotho $(659.3[525.3,827.6]$ vs $787.7[629.5,1007] \mathrm{pg} / \mathrm{ml})$ compared with $\mathrm{MA}^{-}$participants $(p<0.05$ for all; Table 1$)$. Glycaemic control was not significantly different between the two groups. In a multivariate logistic regression analysis, serum soluble Klotho levels were inversely and significantly associated with $\mathrm{MA}^{+}$independent of other variables, including age and eGFR (ESM Table 2). A significant correlation between soluble Klotho and eGFR was not observed in the entire cohort $\left(\mathrm{MA}^{+}\right.$and $\left.\mathrm{MA}^{-}\right)$or $\mathrm{MA}^{+}$population.

No significant association between ACR and soluble Klotho was observed. In participants with $\mathrm{MA}^{+}$(all receiving RAS inhibitors) there was no significant difference in soluble Klotho levels between those with remission of MA $(\mathrm{ACR}<3$ ) vs those with $\mathrm{ACR} \geq 3$.

Other variables including 25-hydroxy vitamin D (measured by liquid chromatography and electrospray ionisation tandem MS), serum corrected calcium (albumin measured by the Roche bromocresol green method and calcium using 5-nitro-5'-methyl-BAPTA method), serum phosphorus (measured using Roche molybdate method) and intact parathyroid hormone (PTH; measured by the Roche method: sandwich assay) levels were available in a subgroup of the full cohort ( $n=30 ; \mathrm{MA}^{+}, n=12 ; \mathrm{MA}^{-}, n=18$ ) (Table 2). These patients were matched for age, $\mathrm{HbA}_{1 \mathrm{c}}, \mathrm{BP}$, diabetes duration and eGFR. In this subgroup, no statistically significant differences were found in 25-hydroxy vitamin D levels, corrected calcium and $\mathrm{PTH}$ between the $\mathrm{MA}^{+}$and $\mathrm{MA}^{-}$groups. Klotho remained significantly lower in participants in the $\mathrm{MA}^{+}$group compared with those in the $\mathrm{MA}^{-}$group. There was a significant negative correlation between serum phosphorus and serum soluble Klotho (Pearson correlation $r=-0.397$, $p=0.03$; ESM Fig. 1).

\section{Discussion}

This is the first study to demonstrate that individuals with type 1 diabetes and $\mathrm{MA}^{+}$have significantly lower serum soluble Klotho levels compared with $\mathrm{MA}^{-}$individuals.

Our findings are consistent with in vivo data showing that Klotho deficiency has a negative impact on albumin excretion and is associated with kidney hypertrophy and exaggerated expansion of the mesangial matrix in renal glomeruli [8]. A nephroprotective role has been demonstrated for Klotho in an animal model of type 1 diabetes, with global overexpression ameliorating the histological features of DKD and reducing albuminuria [9]. In a recent study using a non-diabetic animal model of albuminuria, treatment with exogenous soluble Klotho resulted in a reduction of urinary albumin excretion by protecting against podocyte injury [10].

In individuals with type 2 diabetes, the level of plasma soluble Klotho has been shown to correlate negatively with the development of albuminuria and with a decline in eGFR [11].

Soluble Klotho levels reported in healthy adults, measured with the same assay used in the present study, are similar to our findings in individuals with type 1 diabetes $[5,12]$.
Table 2 Comparison of the clinical and biochemical features, and markers of phosphate balance in a subgroup of 30 type 1 diabetes participants with $\left(\mathrm{MA}^{+}\right)$ and without $\left(\mathrm{MA}^{-}\right)$ microalbuminuria

\begin{tabular}{llll}
\hline Variable & $\mathrm{MA}^{+}(n=12)$ & $\mathrm{MA}^{-}(n=18)$ & $p$ value \\
\hline Age, years & $55.08 \pm 8.8$ & $47.8 \pm 10.7$ & 0.06 \\
Diabetes duration, years & $34.4 \pm 10.4$ & $31.25 \pm 10.44$ & 0.4 \\
$\mathrm{HbA}_{\mathrm{cc}}, \%(\mathrm{mmol} / \mathrm{mol})$ & $7.6(59.43 \pm 6.33)$ & $8.0(63.76 \pm 9.07)$ & 0.32 \\
$\mathrm{eGFR}, \mathrm{ml} / \mathrm{min}$ & $77.2 \pm 28.4$ & $93.9 \pm 24.0$ & 0.09 \\
$\mathrm{ACR}, \mathrm{mg} / \mathrm{mmol}^{\mathrm{a}}$ & $2.4(1,8)$ & $0.75(0.6,1)$ & 0.03 \\
Soluble Klotho, $\mathrm{pg} / \mathrm{ml}^{\mathrm{a}}$ & $674.2(571.8,915.4)$ & $907.3(698.7,1026.1)$ & 0.047 \\
Serum phosphorus, mmol/1 & $1.05 \pm 0.22$ & $0.93 \pm 0.13$ & 0.12 \\
Corrected serum calcium, mmol/1 & $2.36 \pm 0.08$ & $2.35 \pm 0.08$ & 0.6 \\
25-hydroxy vitamin D, nmol/1 & $51.8 \pm 25.2$ & $60.0 \pm 27.0$ & 0.3 \\
Intact PTH, ng/l & $42.8 \pm 31.9$ & $33.1 \pm 16.5$ & 0.3 \\
\hline
\end{tabular}

All data are mean \pm SD unless otherwise stated

${ }^{\text {a }}$ Median (interquartile range) 
Soluble Klotho has antiapoptotic effects on vascular endothelial cells and protects against endothelial dysfunction [2]. The association, if any, between levels of soluble Klotho and CVD or renal endpoints in type 1 or type 2 diabetes is unknown. In non-diabetic elderly individuals, higher soluble Klotho levels are independently associated with a lower risk of CVD [13].

Increased activation of the RAS characterises DKD, whereas treatment with RAS inhibitors lowers the incidence of cardio-renal events in DKD [1]. Of interest, activation of the RAS induces a reduction in serum soluble Klotho levels [2]. We have previously demonstrated in individuals with type 2 diabetes and $\mathrm{MA}^{+}$that RAS inhibition increases soluble Klotho levels [6]. In the present study, despite all participants with $\mathrm{MA}^{+}$receiving RAS inhibitors, this group had significantly lower levels of serum soluble Klotho.

Our work has several limitations. A causal relationship between lower soluble Klotho and development of MA cannot be inferred as this was a cross-sectional study. Our sample size is small as we specifically excluded patients with advanced renal dysfunction and CVD, which are known to influence Klotho levels [2]. We did not measure $24 \mathrm{~h}$ urine excretion of calcium or phosphate or FGF-23 levels, and the results of other markers of calcium phosphate metabolism were only available for around one-third of participants. However, we did not observe any differences in these markers in our study subcohort with relatively preserved eGFR. This is consistent with the general consensus that patients with lower eGFR and advanced DKD exhibit significant perturbations in markers of calcium phosphate metabolism/turnover. In the study subcohort we noted significantly lower levels of circulating Klotho in the $\mathrm{MA}^{+}$group and this may suggest that changes in Klotho occur early in type 1 diabetes DKD and before overt changes in other markers of calcium and phosphorus metabolism.

The strengths of this study are that all of the enrolled patients had relatively preserved renal function (eGFR >45), a similar long duration of diabetes and were a well-characterised cohort attending a single centre for their diabetes care.

Further prospective studies will have to elucidate the contribution of soluble Klotho deficiency to the development of MA and progression of DKD. The need for such studies is underscored by the laboratory evidence that Klotho is involved in the pathogenesis of MA and DKD.

Klotho may be a potential target to reduce or significantly slow the progression of renal disease in individuals with type 1 diabetes. With this perspective, our results establish a platform to address this in future clinical studies.

Data availability The data are available on request from the authors.
Funding This research received no specific grant from any funding agency in the public, commercial or not-for-profit sectors.

Duality of interest The authors declare that there is no duality of interest associated with this manuscript.

Author contribution GM and JK designed the study, interpreted the data and drafted the article. NF analysed the data. RS and LG helped in interpreting the data. All authors reviewed the article and approved the final draft. GM is the guarantor of this work.

Open Access This article is distributed under the terms of the Creative Commons Attribution 4.0 International License (http:// creativecommons.org/licenses/by/4.0/), which permits unrestricted use, distribution, and reproduction in any medium, provided you give appropriate credit to the original author(s) and the source, provide a link to the Creative Commons license, and indicate if changes were made.

\section{References}

1. Karalliedde J, Vibert GC (2011) In: Davies MAS ed. Diabetic nephropathy, 2nd edn. Oxford University Press, Oxford

2. Maltese G, Karalliedde J (2012) The putative role of the antiageing protein Klotho in cardiovascular and renal disease. Int J Hypertens 2012:757469

3. Asai O, Nakatani K, Tanaka T et al (2012) Decreased renal alphaKlotho expression in early diabetic nephropathy in humans and mice and its possible role in urinary calcium excretion. Kidney Int $81: 539-547$

4. Pavik I, Jaeger P, Ebner L et al (2012) Soluble Klotho and autosomal dominant polycystic kidney disease. Clin J Am Soc Nephrol 7: 248-257

5. Pedersen L, Pedersen SM, Brasen CL, Rasmussen LM (2013) Soluble serum Klotho levels in healthy subjects. Comparison of two different immunoassays. Clin Biochem 46:1079-1083

6. Karalliedde J, Maltese G, Hill B, Viberti G, Gnudi L (2013) Effect of renin-angiotensin system blockade on soluble Klotho in patients with type 2 diabetes, systolic hypertension, and albuminuria. Clin J Am Soc Nephrol 8:1899-1905

7. Stevens LA, Schmid CH, Greene T et al (2010) Comparative performance of the CKD Epidemiology Collaboration (CKD-EPI) and the Modification of Diet in Renal Disease (MDRD) study equations for estimating GFR levels above $60 \mathrm{ml} / \mathrm{min} / 1.73 \mathrm{~m}^{2}$. Am J Kidney Dis 56:486-495

8. Lin Y, Kuro-o M, Sun Z (2013) Genetic deficiency of anti-aging gene Klotho exacerbates early nephropathy in STZ-induced diabetes in male mice. Endocrinology 154:3855-3863

9. Kadoya H, Satoh M, Haruna Y, Sasaki T, Kashihara N (2016) Klotho attenuates renal hypertrophy and glomerular injury in Ins2Akita diabetic mice. Clin Exp Nephrol 20:671-678

10. Kim JH, Xie J, Hwang KH et al (2017) Klotho may ameliorate proteinuria by targeting TRPC6 channels in podocytes. J Am Soc Nephrol 28:140-151

11. Kim SS, Song SH, Kim IJ et al (2016) Decreased plasma alphaKlotho predict progression of nephropathy with type 2 diabetic patients. J Diabetes Complicat 30:887-892

12. Yamazaki Y, Imura A, Urakawa I et al (2010) Establishment of sandwich ELISA for soluble alpha-Klotho measurement: agedependent change of soluble alpha-Klotho levels in healthy subjects. Biochem Biophys Res Commun 398:513-518

13. Semba RD, Cappola AR, Sun K et al (2011) Plasma Klotho and cardiovascular disease in adults. J Am Geriatr Soc 59:1596-1601 